\title{
Microscopic Observation of the Intercellular Transport of CdTe Quantum Dot Aggregates Through Tunneling-Nanotubes
}

\author{
Lan Mi ${ }^{1}$, Rongling Xiong ${ }^{1}$, Yu Zhang ${ }^{1}$, Zheng Li $^{1}$, Weidong Yang ${ }^{2}$, Ji-Yao Chen ${ }^{3}$, Pei-Nan Wang ${ }^{{ }^{*}}$ \\ ${ }^{1}$ Key Laboratory of Micro and Nano Photonic Structures (Ministry of Education), Department of Optical Science and Engineering, \\ Fudan University, Shanghai, China; ${ }^{2}$ Department of Biological Sciences, Center for Photochemical Sciences, Bowling Green State \\ University, Bowling Green, USA; ${ }^{3}$ Surface Physics Laboratory (National key laboratory), Department of Physics, Fudan University, \\ Shanghai, China. \\ Email: *pnwang@fudan.edu.cn
}

Received December $9^{\text {th }}, 2010$; revised February $10^{\text {th }}, 2011$; accepted February $14^{\text {th }}, 2011$.

\begin{abstract}
Various inorganic nanoparticles are being considered for applications in life science as fluorescent labels and for such therapeutic applications as drug delivery or targeted cell destruction. It is of importance to understand their intercellular transport behaviors and mechanisms. Here, the intercellular transport of internalized CdTe quantum dot (QD) aggregates through tunneling-nanotubes (TNTs) between human hepatocellular carcinoma cells was studied by time-resolved confocal fluorescence microscopy. TNTs are known to connect eukaryotic cells to provide important pathways for intercellular communications. The formation, shrinkage, elongation and rupture of TNTs were clearly observed by microscopy. We found TNTs contained only F-actin or both microtubules and F-actin. Two transport modes for QD aggregates through the TNTs were observed: the microtubule-based bidirectional motion and the actin-dependent unidirectional motion. The mean square displacement analyses revealed that the intercellular transportations of QDs along TNTs were mediated by active processes. The bidirectional intercellular transport of QDs within lysosomes through the TNT was also observed.
\end{abstract}

Keywords: Quantum Dot, Tunneling-Nanotubes, Active Transport, Fluorescence, Microtubule, Filament

\section{Introduction}

The tunneling-nanotubes (TNTs) were originally described by Gerdes et al for cultured rat neuronal PC12 cells in 2004 [1]. The TNTs are thin tubular protrusions formed from the plasma membranes that connect eukaryotic cells. The lengths of TNTs can reach several cell diameters [1]. They were observed in a variety of cell types both in vivo and in vitro, including the mouse corneal [2], neuronal [1,3], myeloid [4-7], immune [6-11], epithelial [1,12-15] and mast cells [16]. A multitude of cargos, including calcium fluxes [5], [16], bacteria [7], nucleic acids [17], virus [8,11], endosomal vesicles [1,7], lysosomes [3,7], mitochondria [7,17,18], and QDs [19] were observed to be transported through TNTs. Thus, TNTs play an important role in cell-to-cell communication and represent a general mechanism for functional connectivity between living cells. The communications mediated by these long-range physical connections among living cells are more widespread than previously thought.

Recently, a great variety of inorganic nanoparticles (NPs) was synthesized and widely applied in life science as fluorescent labels and in therapeutic applications as drug delivery or targeted cell destruction [20-22]. While intercellular transport can enhance the effectiveness of NP therapeutics, it also potentially results in toxicity to the tissue. Undoubtedly, nanotoxicology has to be matured as a scientific discipline to enable the widespread application of NPs. Hence, it is of great importance to understand the behavior and mechanism of the intercellular transport of NPs.

In the present work, the morphological changes of TNTs and the intercellular transport of the internalized CdTe QD aggregates with relatively larger sizes along the TNTs between human hepatocellular carcinoma (QGY) cells were study by means of differential interference contrast (DIC) imaging and confocal fluores- 
cence microscopy. The QDs are sufficiently bright and photostable for the long term tracking of intercellular events [22,23].

\section{Materials and Methods}

\subsection{Cell Culture}

QGY-7703 cells obtained from the Cell Bank of Shanghai Science Academy were seeded into a Petri dish containing DMEM-H medium with $10 \%$ fetal bovine serum, $100 \mu \mathrm{g} \cdot \mathrm{mL}^{-1}$ streptomycin and $100 \mu \mathrm{g} \cdot \mathrm{mL}^{-1}$ neomycin. The cells were then cultured in a fully humidified incubator at $37^{\circ} \mathrm{C}$ with $5 \% \mathrm{CO}_{2}$ for $24 \mathrm{~h}$.

\subsection{Cell Uptake of QDs and Living Cell Staining}

The water-soluble thiol-capped CdTe QDs with the emission peak at $601 \mathrm{~nm}$ were prepared via the modified hydrothermal route using the thioglycolic acid as a stabilizer [24]. When the cells reached $80 \%$ confluence, the QDs aqueous solution was added into the culture dish to reach a final concentration of $50-100 \mu \mathrm{g} \cdot \mathrm{mL}^{-1}$. The cells were then incubated for $15-30 \mathrm{~min}$ in the incubator for uptake of QDs.

For imaging of lysosomes, the QD loaded cells were further incubated with $50 \mathrm{nM}$ LysoTracker Green DND-26 in growth medium (Invitrogen, Molecular Probes) for another $15 \mathrm{~min}$ to stain lysosomes. After incubation, the Petri dish with the adhered living cells was washed with phosphate-buffered saline (PBS) three times to remove the unbound QDs and fluorescent probes. During the microscopic examination, the cells were kept at $37^{\circ} \mathrm{C} / 5 \% \mathrm{CO}_{2}$ in a temperature controller mounted on the microscope stage (Olympus).

\subsection{Fixation and Immunostaining}

The QGY cells were fixed with $4 \%$ paraformaldehyde (Sigma) for $30 \mathrm{~min}$ at room temperature, and then extracted with $0.1 \%(\mathrm{v} / \mathrm{v})$ Triton X-100 in 4\% paraformaldehyde for $15 \mathrm{~min}$. After washing three times with PBS, the Petri dishes with attached cells were treated with $1 \%$ BSA block for $2 \mathrm{~h}$ at $4^{\circ} \mathrm{C}$, and then incubated in $2 \mu \mathrm{g} \cdot \mathrm{mL}^{-1}$ affinity purified anti- $\alpha$-tubulin (DM1A; eBioscience) overnight at $4^{\circ} \mathrm{C}$. After washing three times with $0.05 \%$ Tween20/PBS, the cells were stained with $2 \mu \mathrm{g} \cdot \mathrm{mL}^{-1}$ R-Phycoerythrin conjugated goat anti-mouse IgG (MultiSciences) for $\alpha$-tubulin labeling and $5-10 \mathrm{nM}$ Alexa Fluor ${ }^{\circledR} 488$ phalloidin conjugate (Invitrogen, Molecular Probes) for F-actin labeling for $2 \mathrm{~h}$ at room temperature. Meanwhile, to reduce nonspecific background staining, $1 \%$ BSA was added to the staining solution. The dishes with attached cells were washed three times with $0.05 \%$ Tween20/PBS before microscopic observation.

\subsection{Microscopy}

The three-dimensional (3-D) fluorescence images and the
DIC micrographs were acquired by a laser scanning confocal microscope (Olympus, FV-300, IX71) using a $488 \mathrm{~nm} \mathrm{Ar}^{+}$laser (MELLES GRIOT) as the excitation source and a $60 \times$ oil objective to focus the laser beam. The fluorescence images of QDs and lysosomes were recorded simultaneously in two channels of the microscope with a 585-640-nm bandpass filter for QDs and a 505-550-nm bandpass filter for lysosomes. Using the t-scan mode (15-180 seconds interval per frame with 2.8-second exposure time for each image) of the microscope, the dynamic morphological change of TNTs and the intercellular transportation of QDs were recorded. Similar method was used to detect the F-actin and microtubes in TNTs. The fluorescence images of F-actin and microtubes were recorded simultaneously in two channels with a 505-550-nm and a 585-640-nm bandpass filter, respectively.

\section{Results and Discussion}

\subsection{TNT Structures}

The QGY cells connected by TNTs were observed in both differential interference contrast (DIC) micrographs and F-actin stained fluorescence images (Figure 1). The TNTs stretched between interconnected cells at their nearest distance. Typically, a seamless transition of the membrane from the TNT to both connected cells was observed, such as those shown in Figure 1(a,b). The junction border between the protrusion of one cell and the membrane of the other connected cell was rarely observed in this work. The DIC image (Figure 1(a)) demonstrates that the protrusions from the two neighboring cells met with each other to form a TNT and the joining point can be seen in the middle of the TNT as marked with an arrowhead. The fluorescence image (Figure 1(c)) shows a 3-D confocal micrograph of the F-actin-stained QGY cells, where the main image exhibits the TNTs in the $X-Y$ plane and the lower part shows an $X-Z$ profile along the TNT in the main image marked with a pair of red arrows. It can be seen clearly that the TNT hovers in the medium and has no contact to the substratum, which is a criterion described for TNTs $[1,25]$. Interestingly, a TNT from a cell branched into two TNTs connecting one nearby cell as shown in Figure 1(d).

Staining for $\alpha$-tubulin and F-actin revealed that TNTs can contain only F-actin (Figure 2(a)) or both microtubules and F-actin (Figure 2(b)). The diameter of the actin-TNT in Figure 2(a) was estimated around $0.4-0.5 \mu \mathrm{m}$, while the microtubule-actin-TNT in Figure 2(b) was relatively thicker with a diameter around $0.7-1 \mu \mathrm{m}$. These results agree with the previously reported observations that microtubules only existed in the thicker TNTs (with a diameter > $0.7 \mu \mathrm{m})$ in human monocyte-derived macrophages [7]. 


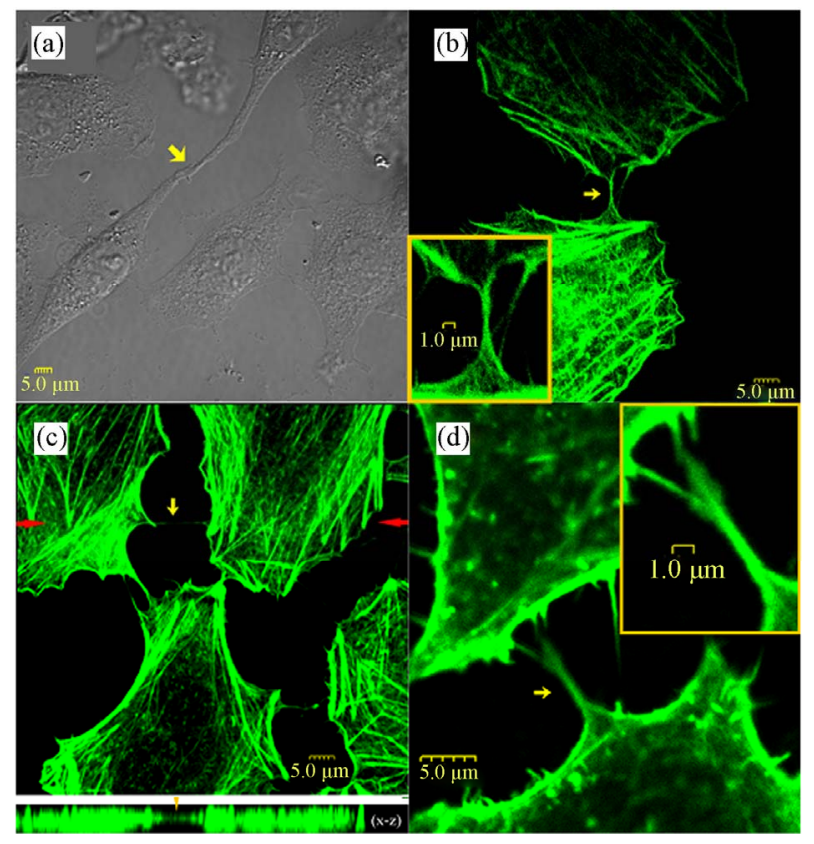

Figure 1. TNTs connecting QGY cells. TNTs are marked with arrowheads in all the images. No junction border between the nanotube and cell surface is observed. (a) DIC images showing the formation of a TNT, where the joining point of the protrusions from the two neighboring cells can be see in the middle of the TNT as marked with an arrowhead. (b-d) Fixed QGY cells were stained with Alexa Fluor ${ }^{\circledR}$ 488 phalloidin (green) to show F-actin contained TNTs. (c) The main image exhibits the TNTs in the X-Y plane and the lower part is an X-Z profile along the TNT in the main image marked with a red arrowhead. (d) A branched TNT connecting two cells. The enlarged views are shown as insets.

The dynamic morphological changes of TNTs and the intercellular transport behaviors of QDs via TNTs were studied using the t-scan mode of the microscope (per frame with 15-180 seconds interval and 2.8-second exposure time). The rupture of a TNT through apparent retraction of the nanotube due to the mechanical stress was demonstrated in Figure 3 (marked with a yellow arrow). A protrusion from a QGY cell reached the membrane of a neighboring cell and the fusion of the membranes at the contact point formed a continuous membrane bridge can be seen in the same figure (marked with a red arrow). Interestingly, the TNT was broken when one of the connected cells died by necrosis as shown in Figure 4.

Both the elongation and shrinkage of TNTs were observed in the time-resolved DIC micrographs. Cell migration was often observed for the adherent QGY cells in the Petri dish. Elongation or shrinkage of a TNT occurred when two TNT-connected cells moving apart or closer. It was suggested that cells maintain a membrane reservoir, which provides a membrane flow into the growing or elongating TNT and draws back the membrane from the shortening TNT [26-28]. However, it seemed that there existed mechanical stress to make TNTs stretched to straight lines with shortest lengths to facilitate the cell-to-cell communication.

\subsection{Transportation of QDs Along TNTs}

The TNTs could also be observed by differential interference contrast (DIC) microscopy as shown in Figure 5. With DIC and fluorescence images acquired by the t-scan mode of the microscope, the intercellular transport behaviors of QDs via TNTs were studied. The watersoluble CdTe QDs were incubated with the QGY cells for 15-30 min. The internalization of QDs was then observed clearly with the 3-D confocal micrographs. The internalized QDs were not diffusely distributed inside the cell. A common observation is that QDs tend to aggregate inside living cells and are often trapped in organelles such as vesicles, endosomes, and lysosomes [29,30]. Microscopic analysis further revealed that the internalized QD aggregates could be transported along TNTs. A series of time-resolved frames selected from a video is shown in Figures 5(b)-(d) to demonstrate the transport of a QD aggregate along the TNT. The size of this QD aggregate was estimated to be about $1.2 \mu \mathrm{m}$, which was in a comparative large size than previously reported cargos $[5,7,8,11,16,19]$. It took 1400 seconds for this QD aggregate to traverse a distance of $4.6 \mu \mathrm{m}$ in the TNT, corresponding to a transport speed of $3.3 \mathrm{~nm} \cdot \mathrm{s}^{-1}$.

Unlike the reported actin-dependent unidirectional transport of cargos through TNTs [1,3,18], our inspections revealed that the QD aggregate changed speed, transiently stopped, or changed direction during its transport along the TNT, corresponding to a bidirectional manner. Figure 5(e) shows its trajectory along the TNT. Since the TNT changed its length during the QD transport, the displacement of the QD aggregate was measured relative to the stationary central point of the TNT. It can be seen clearly that the motion of QDs was bidirectional, in accord with the previous report of the microtubule-based vesicular traffic [7].

Figures 5(f)-(i) show a time-lapse image sequence of two QD aggregates traveling along a TNT towards the cell at the right side. The sizes of these two QD aggregates were estimated to around $0.5-0.7 \mu \mathrm{m}$ in diameter, smaller than the QD aggregate described above in Figures 5(a)-(d). Their moving speeds were measured to be about 28 (yellow arrow) and 21 (green arrow) $\mathrm{nm} \cdot \mathrm{s}^{-1}$, respectively. These transport speeds are much higher than the net transport speed of the QD aggregate $\left(3.3 \mathrm{~nm} \cdot \mathrm{s}^{-1}\right)$ in the bidirectional manner (Figures 5(b)-(d)). By further inspection, it is found that the trajectories of the 


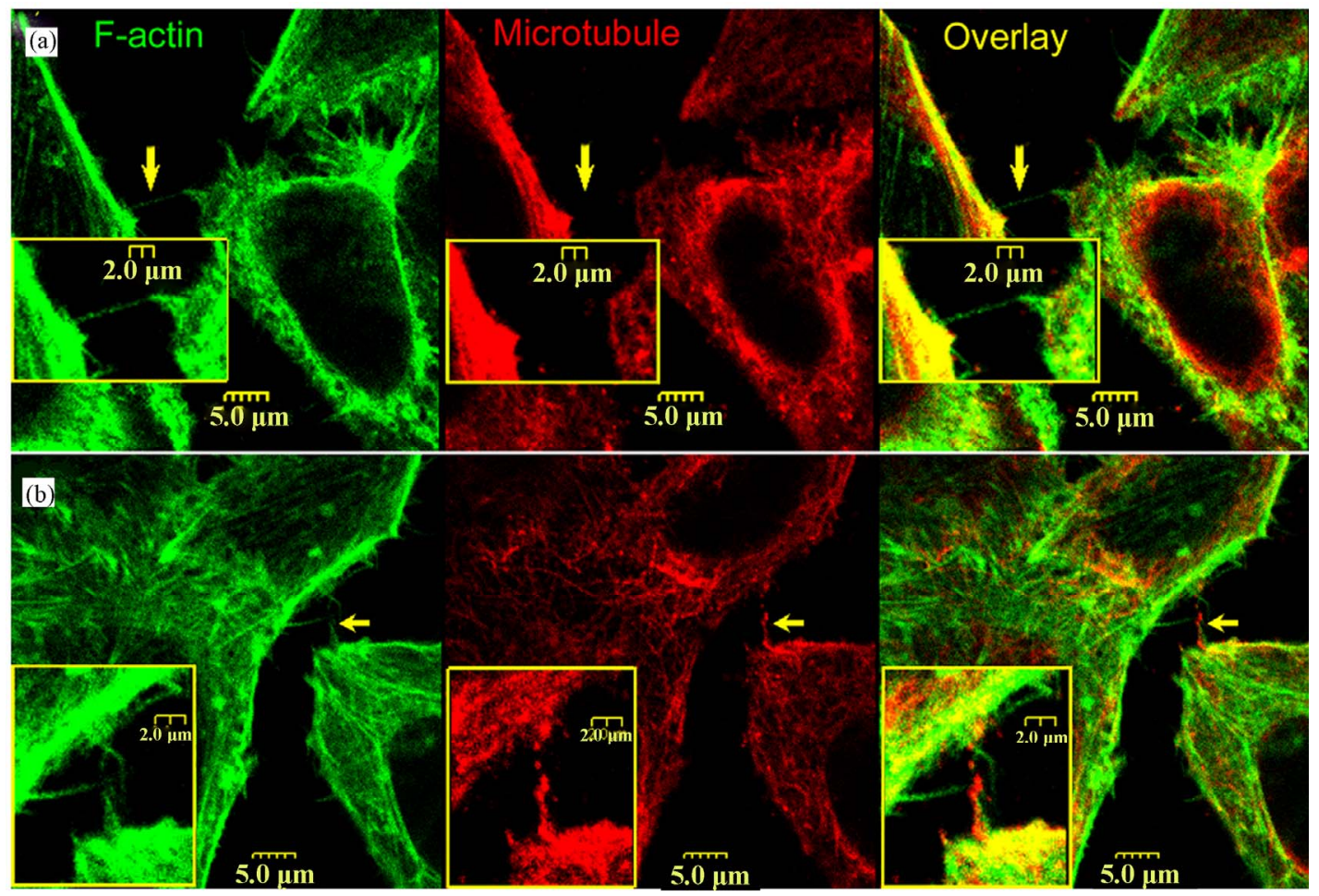

Figure 2. Fixed QGY cells were stained with Alexa Fluor ${ }^{\circledR} 488$ phalloidin for F-actin (green) and immunostained with an antibody against $\alpha$-tubulin (red). (a) A TNT between QGY cells contains F-actin but no microtubule. (b) A TNT between QGY cells contains both F-actin and microtubules. Enlarged views of the TNTs are shown in the insets of the figures. The fluorescence images of $\mathrm{F}$-actin and microtubes were recorded simultaneously in two channels of the microscope with a 505 550-nm and a 585-640-nm bandpass filter, respectively.

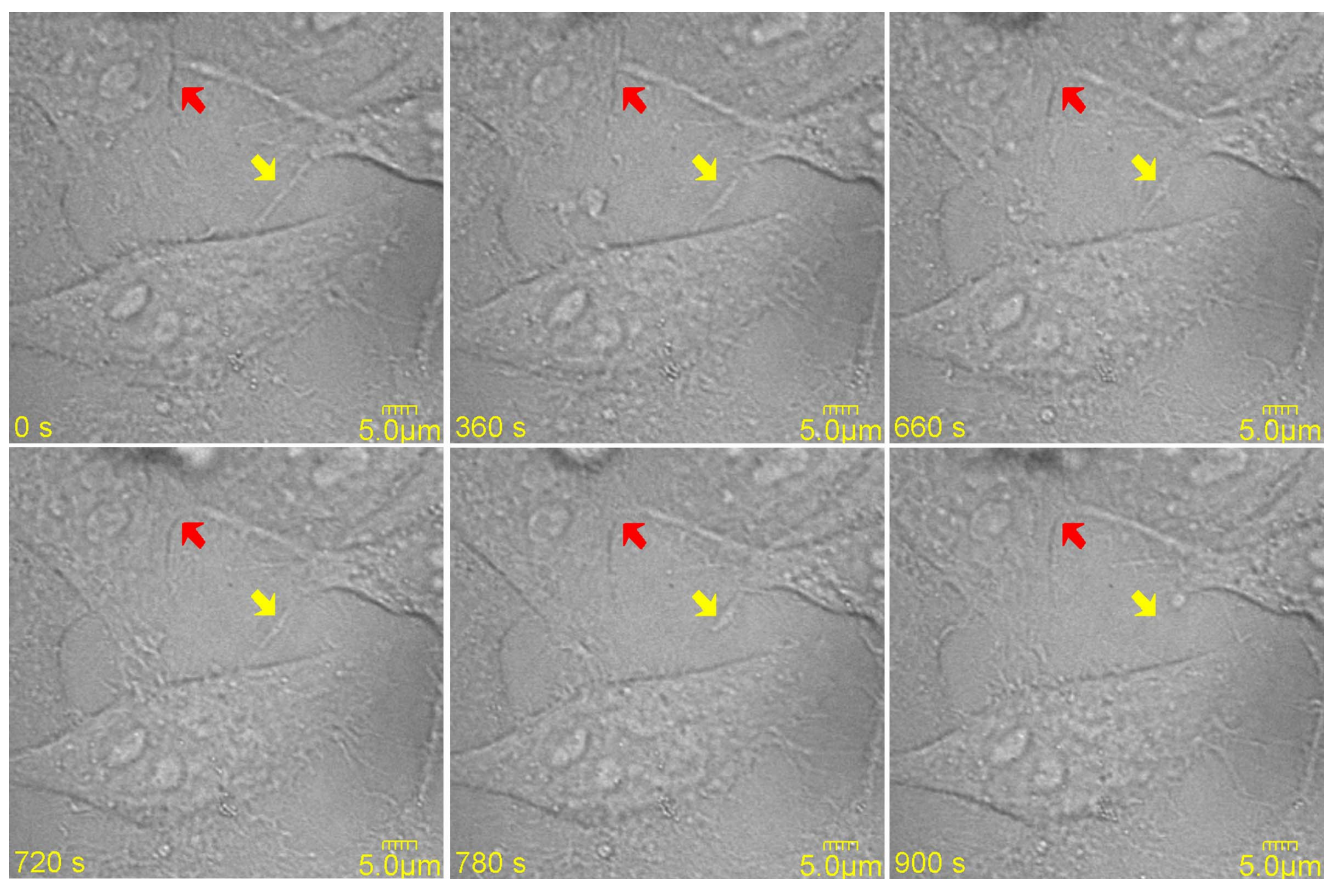

Figure 3. The rupture of a TNT through apparent retraction of the nanotube due to the mechanical stress (marked with a yellow arrow). It can also be seen that a protrusion from a QGY cell reached the surface of a neighboring cell and the fusion of the membranes at the contact point formed a continuous membrane bridge (marked with a red arrow). 


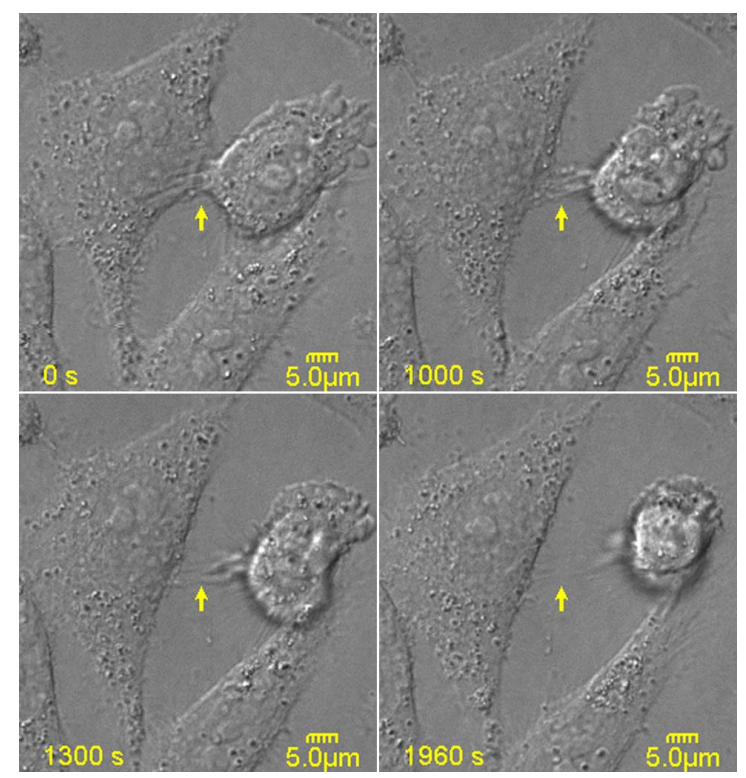

Figure 4. The TNT marked with a yellow arrow was broken when one of the connected cells died by necrosis.
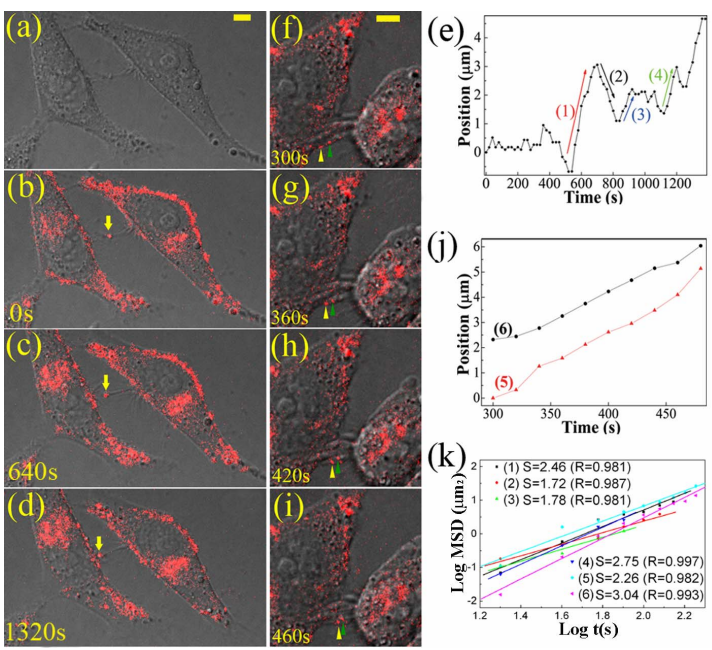

Figure 5. (a) DIC micrograph of two QGY cells connected by a TNT. (b)-(d) Time-lapse merged images of DIC and fluorescence micrographs to show the transport of a QD aggregate (marked with yellow arrowheads) through a TNT. The red color denotes the fluorescence from QDs. (Scale bar, $5 \mu \mathrm{m}$.) (e) Trajectory of the QD aggregate in (b)-(d). Position 0 represents the starting position of the $Q D$ aggregate in the TNT once our measurement began. (f)-(i) Time-lapse images to show two QD aggregates (marked with yellow and green arrows, respectively) traveling along a TNT. (Scale bar, $5 \mu \mathrm{m}$.) (j) Trajectories of the two QD aggregates in (f)-(i), where (5) corresponds to the QD aggregate marked with the yellow arrow and (6) the green arrow. (k) MSD plots for the four periods of continuous moving in (e) that marked as (1), (2), (3) and (4) and the two unidirectional moving in (j) that marked as (5) and (6). The straight lines in the plot are the fitted lines. $S$ represents the slope of the fitted line and $R$ the correlation coefficient. two QD aggregates were unidirectional as shown in Figure $5(\mathrm{j})$, corresponding to the actin-dependent transportations.

When calculating the moving rates of the QD aggregate in the four continuous moving periods in Figure 5(e), it was found that these speeds had the similar value of $18 \pm 4 \mathrm{~nm} \cdot \mathrm{s}^{-1}$, which were much faster than the net speed of the bidirectional motion of the QDs $\left(3.3 \mathrm{~nm} \cdot \mathrm{s}^{-1}\right)$ but were close to the speeds of the unidirectional motions in Figure 5(j) $\left(28\right.$ and $\left.21 \mathrm{~nm} \cdot \mathrm{s}^{-1}\right)$. It means the bidirectional motion greatly reduced the transport speed.

The mean square displacement (MSD) is commonly used to determine whether the movement is active transport or diffusion [13]. The movement can be attributed to the free diffusion when the slope of a $\log -\log$ plot of MSD as a function of time equals 1 , whereas a slope less than 1 refers obstructed movement, and a slope greater than 1 indicates an active transport [31]. As shown in Figure 5(e), there are four periods of the continuous movements. Three of them were forward movements and one backward. By choosing these four continuous movements in Figure 5(e) denoted as (1) to (4) and the two unidirectional movements in Figure 5(j) denoted as (5) and (6), we calculated the relationships of log MSD versus $\log$ t. The results are plotted in Figure 5(k), where the starting time and the displacement of the starting position for every movement were set as 0 . All the simulated slopes (from 1.72 to 3.04) support that the QDs were transported actively along the TNT.

By estimating the size of the QD aggregate and the thickness of the TNT in Figure 5(a), we found that the diameter of the QD aggregate is a little larger than that of the TNT. As reviewed in Ref. [32], this phenomenon was observed previously and called vesicular dilatation. The vesicle-like dilatations of the TNTs may be formed because of an organelle, vesicle or supramolecular assembly with a larger diameter being transported inside them. These dilatations can be transported along the TNT and then released into the cytoplasm of the second cell $[12,33,34]$.

There were two classes of TNTs as shown in Figure 2, which can be distinguished by their cytoskeletal structure and their functional properties. As reported by Onfelt et al, thin TNTs contained only F-actin, whereas thicker nanotubes, i.e., those $>\sim 0.7 \mu \mathrm{m}$ in diameter, contained both F-actin and microtubules [7]. The actin filaments and microtubules are long and directed polymers. They provide the roadways for the cellular transportation system. Mitochondria and intracellular vesicles, including late endosomes and lysosomes, were detected within the thicker TNTs transported in a stepwise and bidirectional manner [7]. 
The bidirectional microtubule-based transport mode has been reviewed by Gross in detail [34]. The transport modes of cargos along TNTs between cells should be same due to the similar cytoskeletal structure. There are three classes of molecular motors which transport cargos along the actin filaments or the microtubules with a certain direction: the myosin motors that move along actin filaments unidirectionally; the kinesin motors that move along microtubules, predominantly towards the microtubule plus-ends; and the dynein motors that move towards the microtubule minus-ends. For microtubulebased transport mode, kinesin and dynein motors can make the cargo move back and forth as a result [34]. The net or average direction of transport depends on which kind of motor plays the dominant role at one moment. For the actin-dependent transport mode, the myosin protein moves the cargo unidirectionally. Figure 6 shows the bidirectional and unidirectional transportations of QDs along a TNT schematically.

\subsection{Transportation of QDs Inside lysosomes Along TNTs}

As reported, a wide variety of intracellular compartments was observed to traffic through the TNTs between macrophages and arrive in the cytoplasm of cells [7]. In our previous work, co-localization of QDs with lysosomes in living cells has been observed [35]. Lysosomes are one of the major destinations of internalized QDs. Hence, it is possible that QDs could transport between cells within lysosomes. To verify this hypothesis, the lysosomes in QGY cells were stained with LysoTracker after the cells were incubated with QDs. The fluorescence images of QDs and lysosomes were recorded simultaneously in two

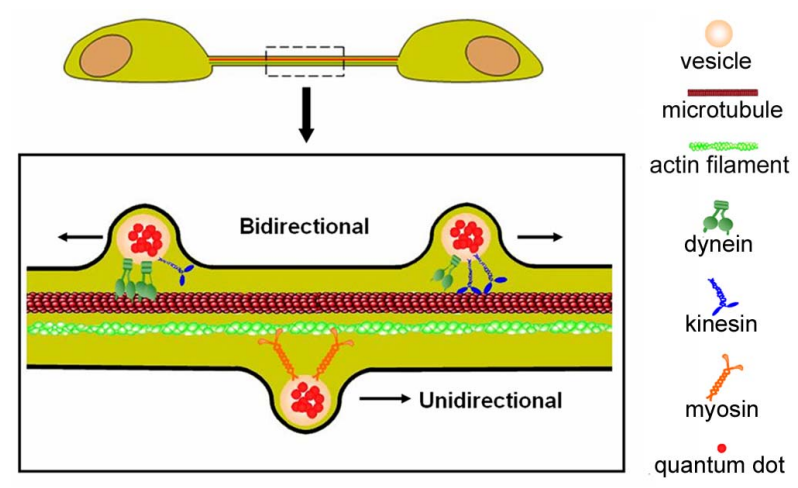

Figure 6. Schematic diagram of possible models for TNTmediated transport of QDs. For microtubule-based transport, the kinesin motors move the cargo along microtubules, predominantly towards the microtubule plus-ends and the dynein motors move the cargo towards the microtubule minus-ends. These two kinds of motors can make the cargo move back and forth as a result. While the myosin motors move the cargo unidirectionally along the actin filament. with a 585-640-nm bandpass filter for QDs and a 505channels of the microscope 550-nm bandpass filter for lysosomes. In the fluorescence micrographs, the red and green colors denote the QDs and the lysosomes, respectively, and the yellow color in the overlaid images represents the mixed fluorescence from LysoTracker and QDs. As shown in Figure 7(a), a QD aggregate was transported along a TNT in the bidirectional manner from the first cell in the upper side of the figure to the second cell at the lower side. After a while, a QD loaded lysosome (yellow color) moved backward along the same TNT to the first cell as shown in Figure 7(b). It means that QDs can transport between cells within or without lysosomes. However, the mechanism should be the same since the internalized QDs are believed to be aggregated inside the endocytotic vesicles.

\section{Conclusions}

TNTs with different diameters were observed to connect QGY cells. The TNTs contained only F-actin or both microtubules and F-actin. The formation, shrinkage, elongation and rupture of TNTs between QGY cells were observed. The internalized QD aggregates were transported to the neighboring cells via TNTs either in a bidirectional or a unidirectional manner. The transport of QDs within lysosomes was also observed. The MSD analyses revealed that the intercellular transportations of QDs along TNTs were mediated by active processes. A fully understanding of the TNT-mediated intercellular transport mechanism for NPs will provide solid fundamental knowledge for the application of NPs in drug delivery.

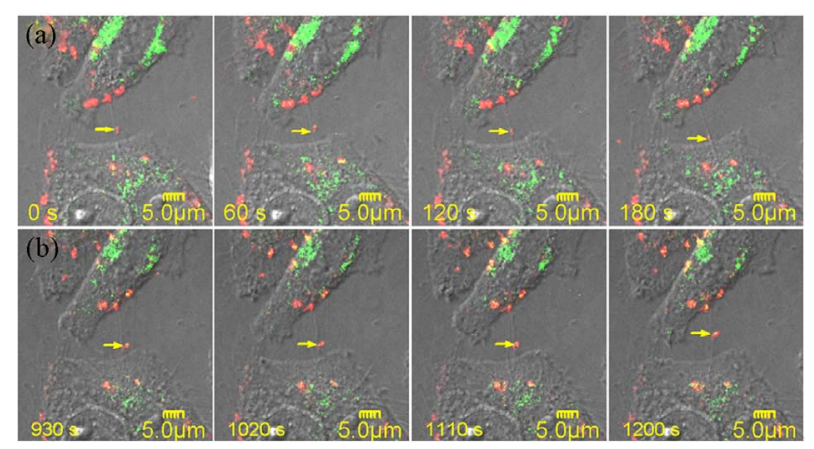

Figure 7. Time-lapse micrographs showing the transport of QD aggregates (marked with yellow arrows) along a TNT between two QGY cells. The red and green colors denote QDs and lysosomes, respectively, and the yellow represents the mixed fluorescence from LysoTracker and QDs, indicating the co-localization of QDs with lysosomes. (a) A QD aggregate was transported along a TNT in the bidirectional manner from the first cell in the upper side to the second cell at the lower side. (b) After a while, a QD loaded lysosome (yellow) moved backward along the same TNT to the first cell. 


\section{Acknowledgements}

This work is supported by National Natural Science Foundation of China $(61008055,11074053)$, the Ph.D. Programs Foundation of Ministry of Education of China (20100071120029) and Shanghai Educational Development Foundation (2008CG03).

\section{REFERENCES}

[1] A. Rustom, R. Saffrich, I. Markovic, P. Walther and H. H. Gerdes, "Notubular Highways for Intercellular Organelle Transport," Science, Vol. 303, No. 5660, 2004, pp. 10071010. doi:10.1126/science. 1093133

[2] H. R. Chinnery, E. Pearlman and P. G. McMenamin, "Cutting Edge: Membrane Nanotubes in Vivo: A Feature of Mhc Class Ii+ Cells in The Mouse Corneal," The Journal of Immunology, Vol. 180, No. 9, 2008, pp. 5779-5783.

[3] K. Gousset, E. Schiff, C. Langevin, Z. Marijanovic, A. Caputo, D. T. Browman, N. Chenouard, F. de Chaumont, A. Martino, J. Enninga, J. C. Olivo-Marin, D. Mannel and C. Zurzolo, "Prions Hijack Tunnelling Nanotubes for Intercellular Spread," Nature Cell Biology, Vol. 11, No. 3, 2009, pp. 328-336. doi:10.1038/ncb1841

[4] D. M. Davis and S. Sowinski, "Membrane Nanotubes: Dynamic Long-Distance Connections between Animal Cells," Nature Reviews Molecular Cell Biology, Vol. 9, No. 6, 2008, pp. 431-436. doi:10.1038/nrm2399

[5] S. C. Watkins and R. D. Salter, "Functional Connectivity between Immune Cells Mediated by Tunneling Nanotubules," Immunity, Vol. 23, No. 2005, pp. 309-318.

[6] B. Onfelt, S. Nedvetzki, K. Yanagi and D. M. Davis, "Cutting Edge: Membrane Nanotubes Connect Immune Cells," Journal of Immunology, Vol. 173, No. 3, 2004, pp. 1511-1513.

[7] B. Onfelt, S. Nedvetzki, R. K. P. Benninger, M. A. Purbhoo, S. Sowinski, A. N. Hume, M. C. Seabra, M. A. A. Neil, P. M. W. French and D. M. Davis, "Structurally Distinct Membrane Nanotubes between Human Macrophages Support Long-Distance Vesicular Traffic or Surfing of Bacteria," Journal of Immunology, Vol. 177, No. 12, 2006, pp. 8476-8483.

[8] S. Sowinski, C. Jolly, O. Berninghausen, M. A. Purbhoo, A. Chauveau, K. Kohler, and et al. , "Membrane nanotubes Physically Connect T Cells over Long Distances Presenting a Novel Route for Hiv-1 Transmission," Nature Cell Biology, Vol. 10, No. 2, 2008, pp. 211-219. doi:10.1038/ncb1682

[9] O. Rechavi, I. Goldstein and Y. Kloog, "Intercellular Exchange of Proteins: The Immune Cell Habit of Sharing," FEBS Letters, Vol. 583, No. 11, 2009, pp. 17921799. doi:10.1016/j.febslet.2009.03.014

[10] D. M. Davis, "Mechanisms and Functions for the Duration of Intercellular Contacts Made by Lymphocytes," Nature Reviews Immunology, Vol. 9, No. 8, 2009, pp. 543-555. doi:10.1038/nri2602
[11] E. A. Eugenin, P. J. Gaskill and J. W. Berman, "Tunneling Nanotubes (Tnt) are Induced by Hiv-Infection of Macrophages: A Potential Mechanism for Intercellular Hiv Trafficking," Cell Immunology, Vol. 254, No. 2, 2009, pp. 142-148. doi:10.1016/j.cellimm.2008.08.005

[12] P. Veranic, M. Lokar, G. J. Schutz, J. Weghuber, S. Wieser, H. Haegerstrand, V. Kralj-Iglic and A. Iglic, "Different Types of Cell-to-Cell Connections Mediated by Nanotubular Structures," Biophysical Journal, Vol. 95, No. 9, 2008, pp. 4416-4425. doi:10.1529/biophysj.108.131375

[13] S. Gurke, J. F. V. Barroso, E. Hodneland, N. V. Bukoreshtliev, O. Schlicker and H. H. Gerdes, "Tunneling Nanotube (Tnt)-Like Structures Facilitate a Constitutive, Actomyosin-Dependent Exchange of Endocytic Organelles between Normal Rat Kidney Cells," Experimental Cell Research, Vol. 314, No. 20, 2008, pp. 3669-3683. doi:10.1016/j.yexcr.2008.08.022

[14] N. M. Sherer, M. J. Lehmann, L. F. Jimenez-Soto, C. Horensavitz, M. Pypaert and W. Mothes, "Retroviruses Can Establish Filopodial Bridges for Efficient Cell-ToCell Transmission," Nature Cell Biology, Vol. 9, No. 3, 2007, pp. 310-315. doi:10.1038/ncb1544

[15] M. Lokar, A. Iglič and P. Veranič, "Protruding Membrane Nanotubes: Attachment of Tubular Protrusions to Adjacent Cells by Several Anchoring Junctions," Protoplasma, Vol. 246, 2010, pp. 81-87. doi:10.1007/s00709-010-0143-7

[16] F. B. Nimita H. Fifadara, Shoichiro Ono and Santa J. Ono, "Interaction between Activated Chemokine Receptor 1 and Fceri at Membrane Rafts Promotes Communication and F-Actin-Rich Cytoneme Extensions between Mast Cells," International Immunology, Vol. 22, No. 2, 2010, pp. 113-128. doi:10.1093/intimm/dxp118

[17] P. Tavi, T. Korhonen, S. L. H. Nninen, J. D. Bruton, S. Lööf, A. Simon and H. Westerblad, "Myogenic Skeletal Muscle Satellite Cells Communicate by Tunnelling Nanotubes," Journal of Cellular Physiology, Vol. 223, No. 2, 2010, pp. 376-383.

[18] M. Koyanagi, R. P. Brandes, J. Haendeler, A. M. Zeiher and S. Dimmeler, "Cell-to-Cell Connection of Endothelial Progenitor Cells With Cardiac Myocytes by Nanotubes," Circulation Research, Vol. 96, 2005, pp. 10391041. doi:10.1161/01.RES.0000168650.23479.0c

[19] K. He, W. Luo, Y. Zhang, F. Liu, D. Liu, L. Xu, L. Qin, C. Xiong, Z. Lu, X. Fang and Y. Zhang, "Intercellular Transfer of Quantum Dots Mediated by Membrane Nanotubes," ACS Nano, Vol. 4, No. 6, 2010, pp. 3015-3022. doi: $10.1021 / \mathrm{nn} 1002198$

[20] B. Fadeel and A. E. Garcia-Bennett, "Better safe than sorry: Understanding the Toxicological Properties of Inorganic Nanoparticles Manufactured for Biomedical Applications," Advanced Drug Delivery Reviews, Vol. 62, No. 3, 2010, pp. 362-374. doi:10.1016/j.addr.2009.11.008

[21] M. Liong, J. Lu, M. Kovochich, T. Xia, S. G. Ruehm, A. E. Nel, F. Tamanoi and J. I. Zink, "Multifunctional Inorganic Nanoparticles for Imaging, Targeting, and Drug Delivery," ACS Nano, Vol. 2, No. 5, 2008, pp. 889-896. 
doi: $10.1021 / \mathrm{nn} 800072 \mathrm{t}$

[22] J. Rao, A. Dragulescu-Andrasi and H. Yao, "Fluorescence Imaging in Vivo: Recent Advances," Current Opinion in Cell Biology, Vol. 18, 2007, pp. 17-25.

[23] J. Gao and B. Xu, "Applications of nanomaterials inside cells," Nano Today, Vol. 4, 2009, pp. 37-51. doi:10.1016/j.nantod.2008.10.009

[24] J. Guo, W. L. Yang and C. C. Wang, "Systematic Study of the Photoluminescence Dependence of Thiol-Capped CdTe Nanocrystals on the Reaction Conditions," Journal Of Physical Chemistry, Vol. 109, 2005, pp. 17467-17473. doi:10.1021/jp044770z

[25] H. H. Gerdes, N. V. Bukoreshtliev and J. F. V. Barroso, "Tunneling nanotubes: A New Route for the Exchange of Components between Animal Cells," FEBS Letter, Vol. 581, No. 11, 2007, pp. 2194-2201. doi:10.1016/j.febslet.2007.03.071

[26] S. Gurke, J. F. V. Barroso and H. H. Gerdes, "The Art of Cellular Communication: Tunneling Nanotubes Bridge the Divide," Histochemistry and Cell Biology, Vol. 129, No. 5, 2008, pp. 539-550. doi:10.1007/s00418-008-0412-0

[27] D. Raucher and M. P. Sheetz, "Characteristics of a Membrane Reservoir Buffering Membrane Tension," Biophysical Journal, Vol. 77, 1999, pp. 1992-2002. doi:10.1016/S0006-3495(99)77040-2

[28] M. Sun, J. S. Graham, B. Hegedüs, F. Marga, Y. Zhang, G. Forgacs and M. Grandbois, "Multiple Membrane Tethers Probed by Atomic Force Microscopy," Biophysical Journal, Vol. 89, 2005, pp. 4320-4329. doi:10.1529/biophysj.104.058180
[29] G. Ruan, A. Agrawal, A. I. Marcus and S. Nie, "Imaging and Tracking of Tat Peptide-Conjugated Quantum Dots in Living Cells: New Insights into Nanoparticle Uptake, Intracellular Transport, and Vesicle Shedding," Journal of the American Chemical Society, Vol. 129, 2007, pp. 14759-14766. doi:10.1021/ja074936k

[30] A. M. Smith, H. Duan, A. M. Mohs and S. Nie, "Bioconjugated Quantum Dots for in Vivo Molecular and Cellular Imaging," Advanced Drug Delivery Reviews, Vol. 60, 2008, pp. 1226-1240. doi:10.1016/j.addr.2008.03.015

[31] M. J. Saxton, "Anomalous Diffusion Due to Obstacles: A Monte Carlo Study," Biophysical Journal, Vol. 66, 1994, pp. 394-401. doi:10.1016/S0006-3495(94)80789-1

[32] M. Lokar, Š. Perutková, V. Kralj-Iglič, A. Iglič and P. Veranič, "Advances in Planar Lipid Bilayers and Liposomes," Elsevier: Burlington, 2009; Vol. 10. doi:10.1016/S1554-4516(09)10003-0

[33] A. Iglič, M. Lokar, B. Babnik, T. Slivnik and P. Veranič, "Possible Role of Flexible Red Blood Cell Membrane Nanodomains in the Growth and Stability of Membrane Nanotubes," Blood Cells Molecules, and Diseases, Vol. 39, 2007, pp. 14-23. doi:10.1016/j.bcmd.2007.02.013

[34] S. P. Gross, "Hither and Yon: A Review of Bi-Directional Microtubule-Based Transport," Physical Biology, Vol. 1, 2004, pp. R1-R11. doi:10.1088/1478-3967/1/2/R01

[35] Y. Zhang, L. Mi, R. Xiong, P.-N. Wang, J.-Y. Chen, W. Yang, C. Wang and Q. Peng, "Subcellular Localization of Thiol-Capped CdTe Quantum Dots in Living Cells," $\mathrm{Na}$ Nanoscale Research Letters, Vol. 4, 2009, pp. 606-612. doi:10.1007/s11671-009-9307-9 